JOURNAL OF SYNCHROTRON RADIATION

ISSN 1600-5775

Received 13 March 2020

Accepted 30 September 2020

Edited by A. F. Craievich, University of São Paulo, Brazil

Keywords: powder X-ray diffraction; high-resolution detectors; multi-analyser detectors; high-energy diffraction; strontium niobate titanate; strontium niobate zirconate; BZT-xBCT.

Supporting information: this article has supporting information at journals.iucr.org/s

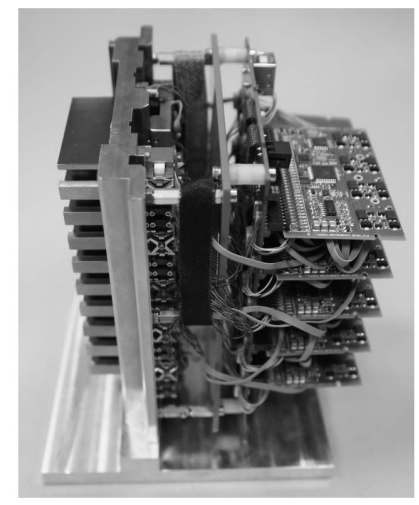

OPEN $\odot$ ACCESS

\section{Multi-analyser detector (MAD) for high-resolution and high-energy powder X-ray diffraction}

\author{
Alexander Schökel, ${ }^{\text {a,b }}$ Martin Etter, ${ }^{b}$ Andreas Berghäuser, ${ }^{c}$ Alexander Horst, ${ }^{\text {d }}$

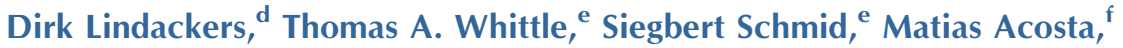 \\ Michael Knapp, ${ }^{a *}$ Helmut Ehrenberg ${ }^{a}$ and Manuel Hinterstein ${ }^{a}$
}

\begin{abstract}
anstitute for Applied Materials, Karlsruhe Institute of Technology (KIT), PO Box 3640, 76021 Karlsruhe, Germany, ${ }^{\mathbf{b}}$ Deutsches Elektronen-Synchrotron (DESY), Notkestrasse 85, 22607 Hamburg, Germany, ${ }^{\mathbf{C}}$ Helmholtz-Zentrum Dresden Rossendorf, FWKX@XFEL, Holzkoppel 4, 22869 Schenefeld, Germany, ${ }^{\mathbf{d}}$ Research Technology, IFW Dresden, PO Box 27 10 16, 01171 Dresden, Germany, ${ }^{\mathbf{e} S}$ School of Chemistry, The University of Sydney, Sydney, NSW 2006, Australia, and f Institute of Materials Science, Technische Universität Darmstadt, Alarich-Weiss-Straße 2, 64287 Darmstadt, Germany. ${ }^{*}$ Correspondence e-mail: michael.knapp@kit.edu
\end{abstract}

For high-resolution powder diffraction in material science, high photon energies are necessary, especially for in situ and in operando experiments. For this purpose, a multi-analyser detector (MAD) was developed for the high-energy beamline P02.1 at PETRA III of the Deutsches Elektronen-Synchrotron (DESY). In order to be able to adjust the detector for the high photon energies of $60 \mathrm{keV}$, an individually adjustable analyser-crystal setup was designed. The adjustment is performed via piezo stepper motors for each of the ten channels. The detector shows a low and flat background as well as a high signal-to-noise ratio. A range of standard materials were measured for characterizing the performance. Two exemplary experiments were performed to demonstrate the potential for sophisticated structural analysis with the MAD: (i) the structure of a complex material based on strontium niobate titanate and strontium niobate zirconate was determined and (ii) an in situ stroboscopy experiment with an applied electric field on a highly absorbing piezoceramic was performed. These experiments demonstrate the capabilities of the new MAD, which advances the frontiers of the structural characterization of materials.

\section{Introduction}

Over recent decades the powder-diffraction technique has developed into one of the most powerful and versatile techniques for structural characterization of materials. It is routinely used in laboratory setups and also in synchrotron and neutron facilities, especially for in situ and in operando experiments (Ehrenberg et al., 2013, 2019). During this development, sophisticated experiments progressively demanded the continuous increase of photon energy (Ehrenberg et al., 2013). Conventional powder-diffraction beamlines usually operate at rather soft photon energies of up to 20-30 keV owing to the low critical energies of the source (Knapp et al., 2004; Patterson et al., 2005; Wallwork et al., 2007; Thompson et al., 2009; Yang et al., 2015; Lausi et al., 2015). For some specific experiments, such as resonant scattering, even energies below $8 \mathrm{keV}$ were used (Staub et al., 2000, 2001; Ehrenberg et al., 2000). For several applications in materials science, such as in situ or in operando studies, energies well above $30 \mathrm{keV}$ are required to be able either to penetrate complete devices or to measure bulk materials with high absorption. A range of beamlines focus on this energy range (Fitch, 2004; Wang et al., 2008; Fauth et al., 2013; Dippel et al., 2015). For total scattering applications, energies between 
50 and $120 \mathrm{keV}$ are typically employed in order to be able to access a large $Q$ range (where $Q$ is the scattering vector) (Shi et al., 2013; Dippel et al., 2015; Sutter et al., 2016; Ren \& Zuo, 2018; Billinge, 2019; Vaughan et al., 2020).

In parallel, detector-development progresses have been made. Typically, the preferred detectors are those exploiting a maximum of the scattered radiation. Although fast acquisition times can be realized by 1D (Rouquette et al., 2012) and area detectors (Daniels et al., 2014) with stroboscopic techniques, they often suffer from limited angular resolution and sensitivity to unwanted stray radiation or background contributions. To achieve angular resolutions at the physical limit, detector concepts using analyser crystals are required. With this concept a decoupling between illuminated sample volume and angular resolution can be achieved. This brings significant drawbacks in measuring only a single point in space at a time, which increases the total measuring time by orders of magnitude. This can be partly compensated by using multianalyser detectors (MADs) with several channels, each with a single-crystal analyser, and recording diffraction patterns simultaneously with a constant $2 \theta$ offset with respect to each other (Hodeau et al., 1998; Gozzo et al., 2004; Toraya, 1996, 2009; Peral et al., 2011; Lee et al., 2008). First proof-of-concept studies using analyser crystals for synchrotron X-ray diffraction experiments were carried out, for example by Buras and Christensen at the DORIS synchrotron in Hamburg (Germany) in 1981 (Buras \& Christensen, 1981). Later implementations by Cox et al. at the Cornell High Energy Synchrotron Source (CHESS) and the Brookhaven National Light Source used the same setup (triple-axis diffractometers equipped with an analyser crystal) to record high-resolution diffractograms (Cox et al., 1983, 1986; Hastings et al., 1984). Similarly, Parrish et al. used an analyser crystal during experiments at the Stanford Synchrotron Radiation Laboratory to improve angular resolution (Parrish et al., 1985, 1986).

Several MAD concepts have been developed in recent years at the Photon Factory (PF) (Toraya et al., 1996), the European Synchrotron Radiation Facility (ESRF) (Hodeau et al., 1998; Dejoie et al., 2018), the Swiss Light Source (SLS) (Gozzo et al., 2004), the ALBA synchrotron (Peral et al., 2011) and the Advanced Photon Source (APS) (Lee et al., 2008). The common design of a typical MAD covers photon energies between 8 and $40 \mathrm{keV}$. In order to adjust the analyser crystals for the different energies and maximize the transmitted intensities, different levels of complexity have been developed. The simplest design allows a degree of freedom of the secondary collimator and the scintillator detectors with respect to the analyser crystals and the crystals itself (Gozzo et al., 2004; Hodeau et al., 1998). More sophisticated solutions were developed (Peral et al., 2011) or include even individually adjustable analyser crystals (Lee et al., 2008). These additional degrees of freedom are implemented in order to optimize signal-to-noise ratio and diffracted intensity, since the beam paths on the off-centre crystals and through the channels follow non-linear pathways. A clever approach to optimize these dependencies was proposed by Peral et al. (2011) with a Rowland circle construction. With this setup the disadvantages of beam walk across the components are minimized. A detailed treatment of the instrumental resolution function (IRF) in the presence of mirrors and analysers can be found in the work of Gozzo et al. (2006). However, MAD designs for a broad energy range are limited to maximum photon energies of $\sim 40 \mathrm{keV}$. For higher energies, the diffraction angles become smaller and the separation of channels is more challenging and cannot be combined with the adjustment for low photon energies. However, sophisticated in situ or in operando experiments in materials science or for fundamental research, together with complex sample environments or transmission geometry setups, are frequently limited by absorption. Since the ideal ratio between absorption and sample thickness is reached at $\mu R=1$, with $\mu$ being the absorption coefficient and $R$ being the sample radius, complex sample environments or geometries can only be realized with increasing photon energy and thus decreasing $\mu$ (Cullity \& Stock, 2001; Ehrenberg et al., 2013, 2019). These experiments demand higher photon energies with a higher penetration depth or higher transmission capability (Schmitt et al., 2013; Ehrenberg et al., 2019).

The challenge with a MAD setup for high photon energies is the small Bragg angles owing to the need to separate the beam paths inside the detector for the direct and the diffracted beams. This channel crosstalk becomes critical for photon energies above a certain limit. As an example, the Si 111 reflection which is used for analyser crystals exhibits a diffraction angle of $\theta=1.888^{\circ}$ at $60 \mathrm{keV}$. A typical diffraction pattern usually recorded up to $2 \theta=90^{\circ}$ using $\mathrm{Cu} K \alpha_{1}$ radiation shrinks to a $2 \theta$ range of only $10.5^{\circ}$ for this photon energy of $60 \mathrm{keV}$. This requires sophisticated shielding, fine adjustment of the analyser crystals and a sufficiently small angular step width of data points. The required step width is determined by the available angular resolution that comes along with the Darwin width of a Si 111 reflection of $2.4 \times 10^{-4}$ degrees. Furthermore, the high penetration capability of the $60 \mathrm{keV}$ primary beam energy requires a proper shielding of scintillator detectors and the collimator path in order to maximize the signal-to-noise ratio.

In order to combine experiments with high penetration capability for highly absorbing materials or in situ experiments with high angular resolution and to overcome the limitations of ordinary powder-diffraction experiments, specialized approaches are necessary. One way is to select a photon energy which is high enough to not be limited by absorption edges. In this case, no fluorescence effects limit the diffraction experiments. Such energies typically lie in the range of $\sim 60 \mathrm{keV}$ and above. On the other hand, the energies should be low enough to be able to be handled with reasonable effort in terms of shielding with tungsten- or tantalum-based materials. A photon energy of $60 \mathrm{keV}$ constitutes a reasonable compromise between the cases described and is generally suited for the majority of specifications.

In this article, we present a ten-channel MAD for the powder-diffraction side station P02.1 (Dippel et al., 2015; Herklotz et al., 2013) at the PETRA III storage ring of the Deutsches Elektronen-Synchrotron (DESY) in Hamburg, 
Germany. This side station operates with an undulator as a radiation source at a fixed energy of $60 \mathrm{keV}$ and is dedicated to high-resolution powder diffraction for material science applications (Liu et al., 2017; Liu, Knapp, Ehrenberg et al., 2016; Liu, Knapp, Schmitt et al., 2016; Hinterstein et al., 2018), in situ and in operando studies (Schader et al., 2016; Geiger et al., 2017, 2018; Mgbemere et al., 2017; Hinterstein et al., 2019; Riess et al., 2019; Lee, Shi, Kumar, Hoffman, Etter, Checchia, Winter et al., 2020; Lee, Shi, Kumar, Hoffman, Etter, Winter et al., 2020; Choe et al., 2015), and total-scattering experiments (Yavuz et al., 2015). Together with proper synchronization with a periodic excitation, this detector can also be used for high-resolution in situ and in operando stroboscopic measurements on materials under the influence of external stimuli (Liu et al., 2020; Lee, Shi, Kumar, Hoffman, Etter, Winter et al., 2020; Choe et al., 2015). The capability of this detector at beamline P02.1 is shown with measurements on different reference materials [e.g. NIST 660a/b (the National Institute for Standards and Technology), NIST 640d, NIST 674b]. The in situ behaviour of a highly absorbing piezoceramic and the elucidation of the complex structure of strontium niobium titanate were also determined.

\section{Technical realization}

The standard detector setup for the beamline is a Perkin Elmer area detector with an active area of $409.6 \mathrm{~mm} \times$ $409.6 \mathrm{~mm}$ and a pixel size of $200 \mu \mathrm{m}^{2}$. For accurate profileshape measurements or powder-diffraction measurements at the resolution limit, the ten-channel MAD can alternatively be moved in and rotated around the sample, which itself is mounted in the diffractometer centre. The technical layout of the detector is mainly based on tungsten alloy (Densimet) collimators. Since the beamline is operated at a fixed photon energy, no complex adjustment mechanism is necessary for the analyser crystals. Owing to the high photon energy, the necessary $2 \theta$ scanning range is rather small. Therefore, the channels were designed with a separation of just $1^{\circ}$ in $2 \theta$ from each other. This requires accurate shielding and channel separation for the direct and the diffracted beam in the collimators. To maximize the diffracted intensity and the signal-to-noise ratio, the analyser crystals have to be adjusted accurately. Even miscuts of the crystals and small misalignments can already lead to a significant decrease in diffracted intensity. The alignment of the analyser-crystals' angle was originally performed via stepper-motor-driven spindles sitting on a slide and acting on a lever arm (Horst et al., 2013). However, owing to stability reasons and higher repeatability and accuracy, linear piezo actuators with magnetic encoder and reference markers were used to act via lever arms on the crystals. The intensity diffracted by the analyser crystals was detected via scintillation counters (see Fig. S1 in the supporting information).

The whole detector sits on a supporting aluminium baseplate where all components can be pre-aligned in machined seats (Fig. 1). The main components are first collimator unit, crystal unit, second collimator unit and supporting aluminium

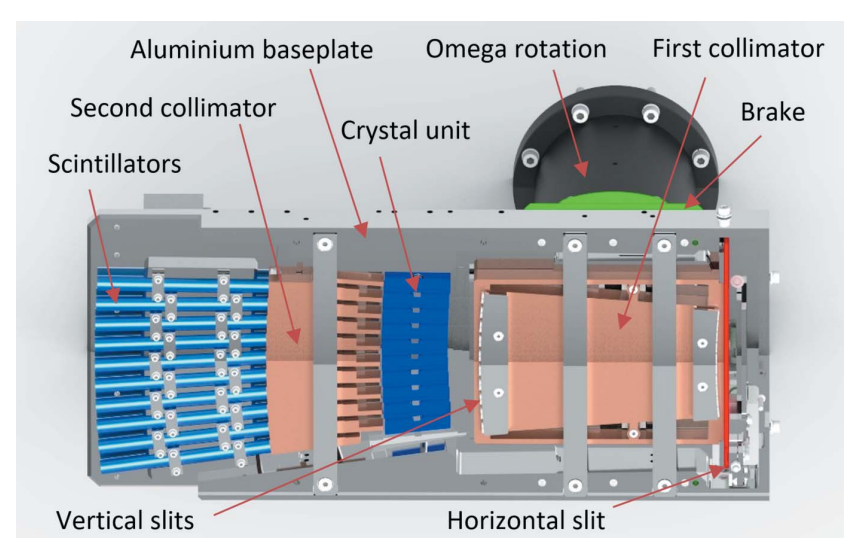

Figure 1

A schematic overview of the complete MAD.

baseplate with scintillator holders. The first and second collimator units are machined with spark erosion from a monolithic block of tungsten alloy (Densimet) to avoid crosstalk between the individual channels and to block fluorescence that is created along the beam path (Fig. S2). Although the incoming primary beam is highly collimated, it turns out that fluorescence is a serious issue, especially for the channels in the lower part of the detector close to the primary beam path. The individual channels have an acceptance angle of $3.27^{\circ}$ that can be further narrowed down with slits on the entrance and exit of the first collimator to $0.73^{\circ}$. These slits can also be replaced by custom-made pin diodes for initial alignment (Fig. S3). In front of the detector entrance, two vertical blades act as a horizontal slit to limit the accepted beam in the plane perpendicular to the diffraction plane. The whole setup can be rotated by a stepper motor (omega rotation) for initial angular alignment and the post with the detector attached is finally being blocked by a brake.

The analyser crystals are $55 \mathrm{~mm}$-long $\mathrm{Si}(111)$ crystals and all axes of rotation sit in a common Densimet block exactly around the circumference of the detector circle. To ensure high accuracy and reproducibility of the rotation, and since the required angular range is only about $\pm 0.5^{\circ}$, a flexure hinge bearing was used for the axes (Fig. 2). One half axis is glued to the front side of the crystal with an opening large enough to let

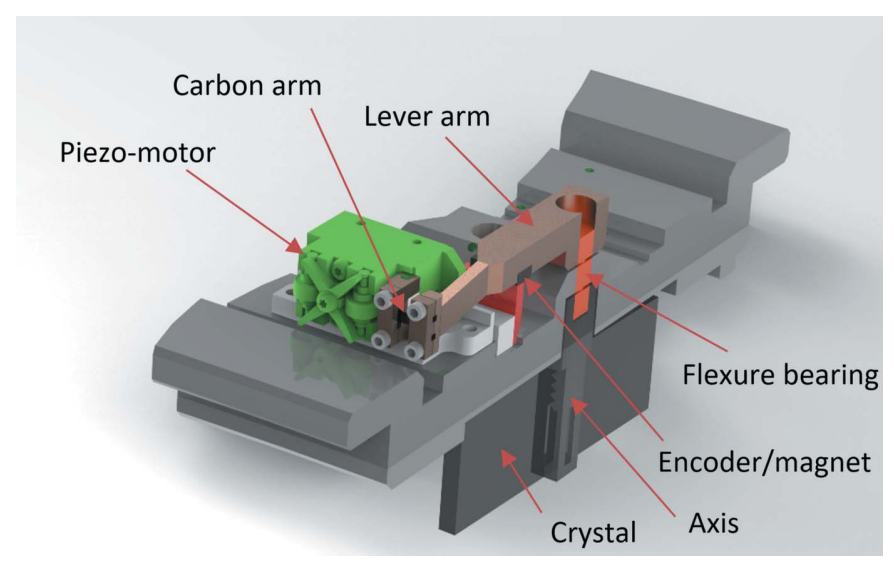

Figure 2

A detailed view of a single piezo-motor-based crystal-alignment module. 

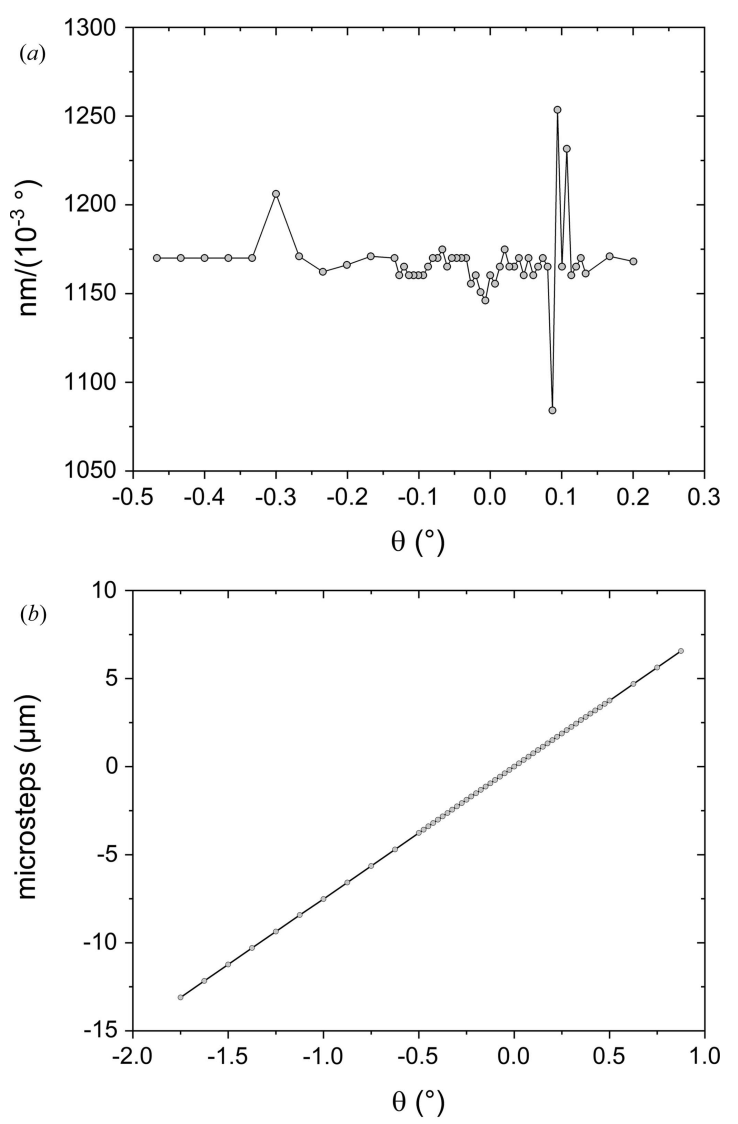

Figure 3

Plots of $(a)$ piezo-motor reproducibility versus analyser-crystal axis position and $(b)$ piezo-motor microsteps versus analyser-crystal deflection, measured with an autocollimator.

the beam pass through. The second half axis has a lever arm mechanism that is elastically fixed to a linear piezo-motor drive. Both half axes are fitted into the flexure hinge. The elastic connection between the lever arm and the linear piezo drive is made of carbon-fibre-enforced polymer. The linear piezo motor has a magnetic encoder system with reference position. The linear resolution per microstep was chosen to be $7.8 \mathrm{~nm}$ which transfers into $2.5 \times 10^{-5}$ degrees. Fig. 3(a) shows the reproducibility of the crystal angle measured with an interferometer versus piezo-motor microsteps. These results over a tilting range of $2.5^{\circ}$ show an average value of $\sim 1175 \mathrm{~nm}\left(10^{-3} \text { degrees }\right)^{-1}$, which results in $\sim 150$ steps $\left(10^{-3} \text { degrees }\right)^{-1}$. The maximum deviation is $\sim 100 \mathrm{~nm}$ $\left(10^{-3} \text { degrees }\right)^{-1}$. For the linear plot of microsteps versus analyser-crystal deflection, this deviation is negligible and the setup shows a smooth straight line [Fig. 3(b)].

The positioning of each analyser crystal is controlled by custom-built electronic boards [Fig. 4(a)] which allow a closedloop regulation for the piezo motor in a range down to the resolution limit, which corresponds to a resolution of $1.6 \times 10^{-5}$ degrees for the crystal. A ten-channel multiplexer circuit connects each piezo-motor channel via USB or serial port to the remote computer. Comprehensive tests with the direct beam at beamline P02.1 with $60 \mathrm{keV}$ showed that even small corrections in the closed-loop mode can be seen in the diffracted-beam profile. To avoid this, we used the closed-loop positioning system only for the primary adjustment procedure of the crystal. Once the maximum count rate is reached, the proportional-integral-derivative (PID) controller can be switched off and the piezo motor is forced via a command to settle down into a 'parking position'. The position of the motor is held by mechanical clamping and the electronic voltage supply can be switched off.

\section{Performance}

The performance of the MAD was determined by measurements of the attenuated primary beam and by measurements of commercially available powder X-ray diffraction standards obtained by NIST. The long-term stability of each channel regarding zero shift and integrated intensities was determined by primary beam measurements and can be found in the supporting information.

For the determination of the IRF, several NIST powder $\mathrm{X}$-ray diffraction reference standards were measured: $\mathrm{LaB}_{6}$ (NIST 660a, NIST 660b), silicon (NIST 640d) and $\mathrm{CeO}_{2}$ (NIST 674b). The measurements were taken on different days. All measurements were performed in a continuous scan mode (sweep mode) for the $2 \theta$ circle (see the supporting informa-

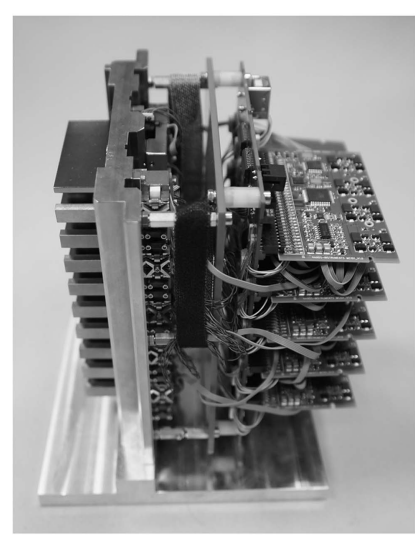

(a)

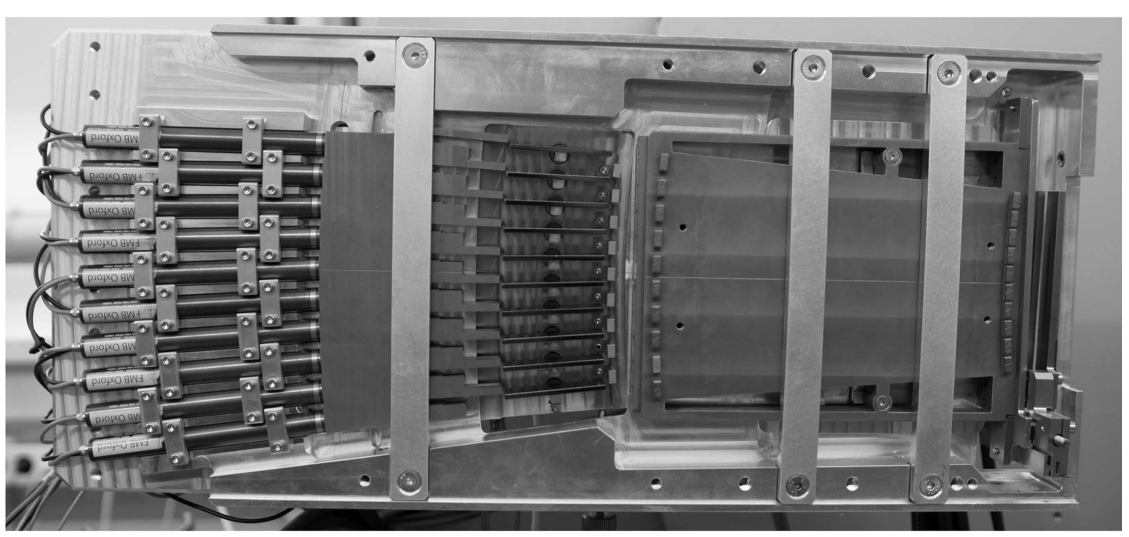

(b)

Figure 4

(a) An analyser block with electronics and (b) the MAD mounted on a diffractometer with the protective cover removed. 


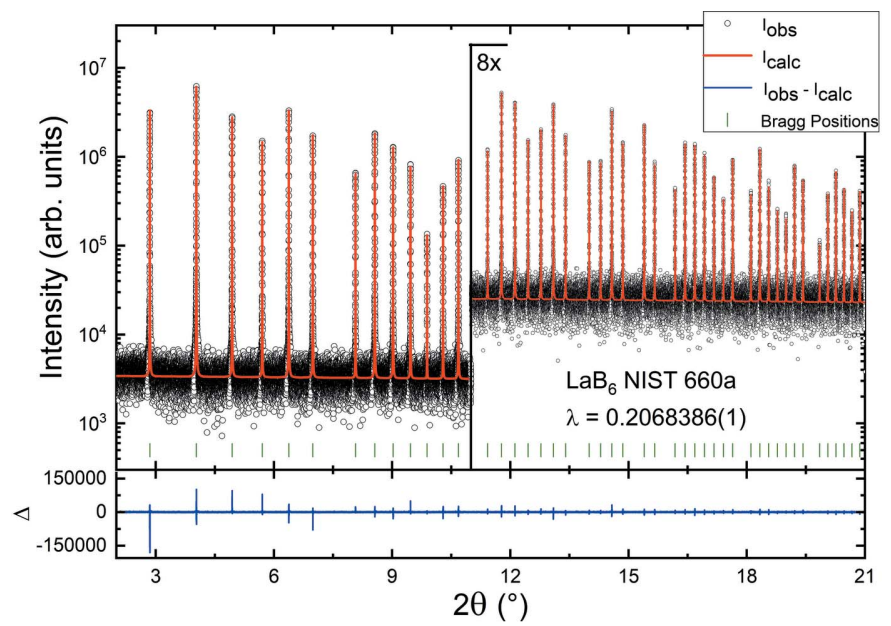

(a)

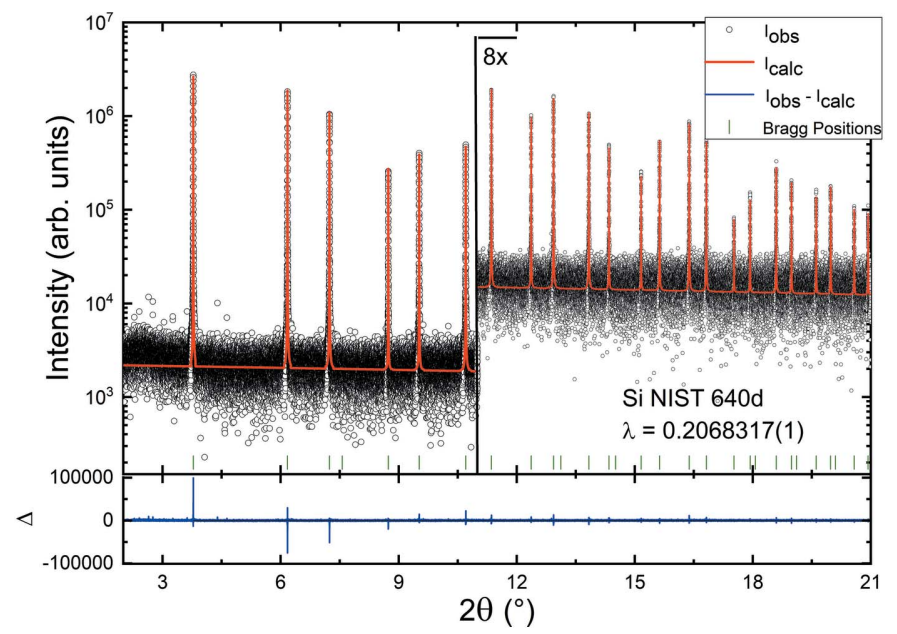

(c)

Figure 5

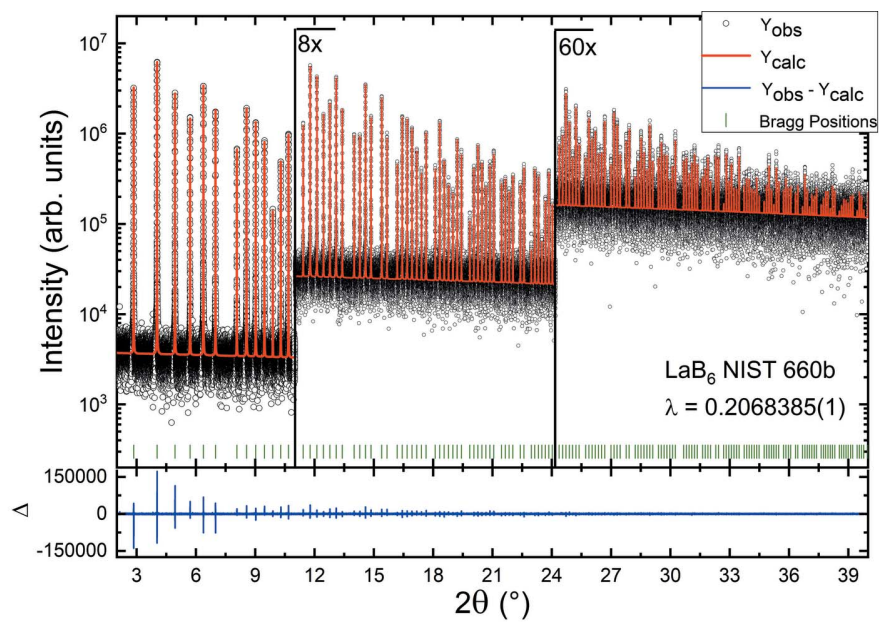

(b)

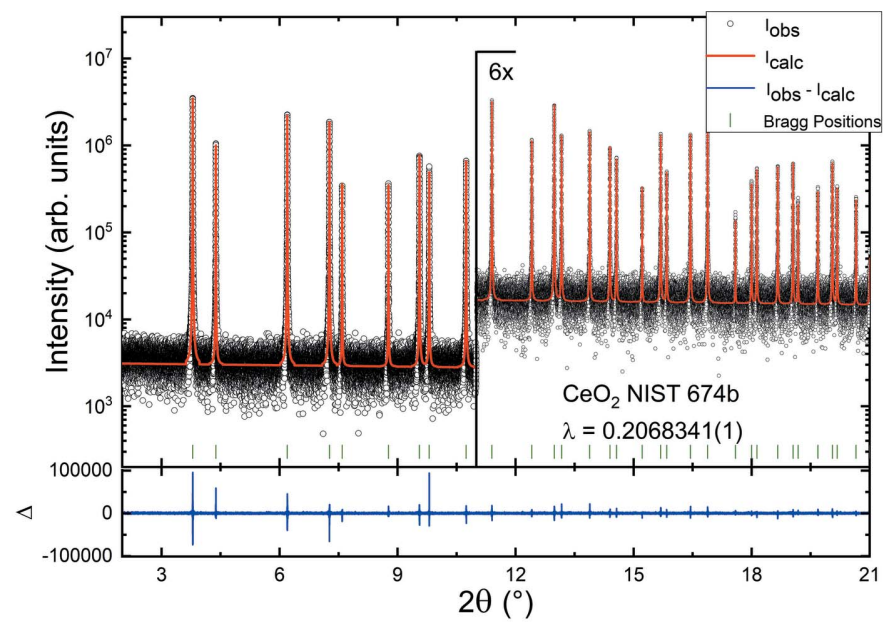

(d)

Powder X-ray diffraction measurements with the MAD (circles) and corresponding Pawley refinements (red line) of NIST standards: (a) LaB ${ }_{6}$ NIST 660a, (b) $\mathrm{LaB}_{6}$ NIST 660b, (c) silicon NIST 640d and (d) $\mathrm{CeO}_{2}$ NIST 674b. Difference curves below each diffraction pattern are enhanced.

tion) with a step width after re-binning of $2.4 \times 10^{-4}$ degrees. Measurements of the individual channels during one run were subsequently merged into a single diffraction pattern. Pawley refinements (Pawley, 1981) of these measurements made with the program TOPAS (Coelho, 2018) can be found in Figs. $5(a)-5(d)$.

Refined parameters from all Pawley fits can be found in Table 1. For all refinements the Thompson-Cox-Hastings pseudo-Voigt approach (Thompson et al., 1987) for modelling the $2 \theta$-dependent reflection profiles was used, plus the model of Finger et al. (1994) in order to account for the asymmetric axial divergence effect (the algorithm models the shifts and low-angle tails for peaks below $\sim 2 \theta=30^{\circ}$ ). During the process of refinement, different axial divergence models were initially tested and it turned out that the 'simple axial model' implemented in the TOPAS software could be used in order to obtain an adequate modelling of the asymmetric reflection profiles. However, by changing to the model of Finger et al. (1994) with parameters fixed to their physical values $(L=$ $551.33 \mathrm{~mm}, S=0.8 \mathrm{~mm}, H=2.65 \mathrm{~mm}$ ) a slightly improved fit could be obtained in terms of a flatter difference curve and a reduced weighted profile $R$ factor [residual factors and the goodness of fit $(\mathrm{GoF})$ are used as defined in the TOPAS program (Coelho, 2018)]. For each Pawley refinement, the wavelength, two background coefficients and five ThompsonCox-Hasting parameters were refined. The quality of the obtained IRF was verified by plotting these functions together with the full width at half-maxima (FWHM) determined by pseudo-Voigt fits of individual reflections of all reference materials (Fig. 6). All the determined IRFs are in good agreement with the individually fitted reflection widths and behave as described by Masson et al. (2001).

Although the obtained parameters and fits are almost in perfect accordance with expected values, we note that the values for the GoF as well as the (weighted) profile $R$ values (even the background-corrected ones) are much higher than one would expect from the appearance of the refinements. These high $R$ values can be explained by taking the low background into account, which is in fact flat over the entire measurement range, but showing a rather high scattering, the reason of which could not be identified. When normalizing the highest reflection in the diffraction pattern of Fig. 5(a) to 100, 
Table 1

An overview of the determined parameters from Pawley refinements.

Residual factors and the GoF were obtained as defined in the TOPAS program (Coelho, 2018). Lattice parameters are fixed to the NIST certificate values (certified at $22.5^{\circ} \mathrm{C}$ ). The measurements were performed at $23.0^{\circ} \mathrm{C}$.

\begin{tabular}{|c|c|c|c|c|}
\hline $\begin{array}{l}\text { Material } \\
\text { Standard }\end{array}$ & $\begin{array}{l}\mathrm{LaB}_{6} \\
\text { NIST 660a }\end{array}$ & $\begin{array}{l}\mathrm{LaB}_{6} \\
\text { NIST 660b }\end{array}$ & $\begin{array}{l}\mathrm{Si} \\
\text { NIST 640d }\end{array}$ & $\begin{array}{l}\mathrm{CeO}_{2} \\
\text { NIST 674b }\end{array}$ \\
\hline Capillary & Kapton tube, $0.8 \mathrm{~mm}$ diameter & & & \\
\hline Wavelength $(\AA)$ & $0.2068386(1)$ & $0.2068385(1)$ & $0.2068317(1)$ & $0.2068341(1)$ \\
\hline Lattice parameter $(\AA)$ & 4.1569162 & 4.15689 & 5.43123 & 5.411526 \\
\hline$U$ & $8.04(10) \times 10^{-4}$ & $7.72(8) \times 10^{-4}$ & $4.90(31) \times 10^{-4}$ & $8.05(34) \times 10^{-4}$ \\
\hline$V$ & $-1.66(12) \times 10^{-5}$ & $-2.08(11) \times 10^{-6}$ & $-1.20(38) \times 10^{-5}$ & $-6.96(56) \times 10^{-5}$ \\
\hline$W$ & $3.92(34) \times 10^{-7}$ & $9.72(4) \times 10^{-7}$ & $1.61(110) \times 10^{-7}$ & $6.18(22) \times 10^{-6}$ \\
\hline$Z$ & 0 & 0 & 0 & 0 \\
\hline$X$ & $2.52(8) \times 10^{-3}$ & $2.50(8) \times 10^{-3}$ & $17.03(27) \times 10^{-3}$ & $11.35(21) \times 10^{-3}$ \\
\hline$Y$ & $3.13(5) \times 10^{-4}$ & $4.25(5) \times 10^{-4}$ & $2.98(16) \times 10^{-4}$ & $18.69(15) \times 10^{-4}$ \\
\hline$\mu R($ calculated $) \dagger$ & 0.56 & 0.56 & 0.01 & 1.40 \\
\hline$R_{\exp }$ & 1.12 & 1.31 & 1.67 & 1.14 \\
\hline$R_{\exp }^{\prime}$ & 1.47 & 1.89 & 2.46 & 1.46 \\
\hline$R_{\mathrm{p}}$ & 10.98 & 13.56 & 17.49 & 10.59 \\
\hline$R_{\mathrm{p}}^{\prime \prime}$ & 16.77 & 23.54 & 31.09 & 15.45 \\
\hline$R_{\mathrm{wp}}$ & 17.24 & 20.58 & 26.39 & 17.78 \\
\hline$R_{\mathrm{wp}}^{\prime \prime}$ & 22.57 & 29.59 & 38.91 & 22.79 \\
\hline$R_{\mathrm{B}}$ & 1.06 & 1.03 & 0.72 & 0.58 \\
\hline $\operatorname{GoF}\left(R_{\mathrm{wp}} / R_{\mathrm{exp}}\right)$ & 15.36 & 15.67 & 15.80 & 15.64 \\
\hline $\operatorname{GoF}\left(R_{\mathrm{B}} / R_{\mathrm{exp}}\right)$ & 0.95 & 0.79 & 0.43 & 0.51 \\
\hline
\end{tabular}

$\dagger \mu R$ is calculated with the help of https://11bm.xray.aps.anl.gov/absorb/absorb.php assuming a packing fraction of 0.5 .

the highest average background value is $\sim 0.07 \pm 0.04$ for low angles and decreases linearly to $0.02 \pm 0.02$. Considering the high photon energy and hence the greater difficulties in controlling stray radiation, the signal-to-background ratio is excellent. With very sharp reflections, even for conservative calculations, this results in just $1 \%$ of the data points containing information about reflections. Therefore, the relative scattering of the background data points contributes significantly to the seemingly inferior refinement parameters.

\section{Materials science case studies}

In the following, two scientific examples are shown that demonstrate the capabilities of the MAD at the P02.1 beam-

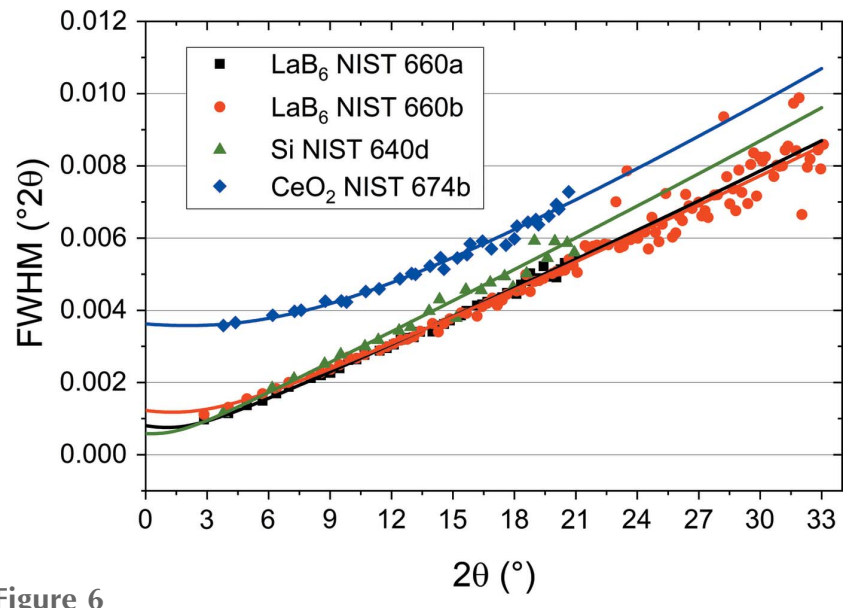

Figure 6

FWHM determined for individual reflections of $\mathrm{LaB}_{6}$ NIST 660a (squares), $\mathrm{LaB}_{6}$ NIST 660b (circles), silicon NIST 640d (triangles) and $\mathrm{CeO}_{2}$ NIST $674 \mathrm{~b}$ (diamonds). The corresponding lines were calculated using the Thompson-Cox-Hasting parameters, which were determined from Pawley refinements. line. Firstly, we provide a case study using strontium niobate titanate $\left(\mathrm{Sr}_{3} \mathrm{TiNb}_{4} \mathrm{O}_{15}, \mathrm{STN}\right)$ and strontium niobate zirconate $\left(\mathrm{Sr}_{3} \mathrm{ZrNb}_{4} \mathrm{O}_{15}, \mathrm{SZN}\right)$. We demonstrate that former unresolved structural features can be investigated in complex crystal structures. The combination with the $2 \mathrm{D}$ detector illustrates the capabilities for the identification of secondary phases in functional materials. We further provide a second case study using 0.6 $\mathrm{BaZr}_{0.2} \mathrm{Ti}_{0.8} \mathrm{O}_{3}-0.4 \mathrm{Ba}_{0.7} \mathrm{Ca}_{0.3} \mathrm{TiO}_{3}$ (BCZT) and an in situ stroboscopic investigation of the field-induced processes. This case study demonstrates the need for a combination of high photon energies for in situ or in operando experiments in transmission geometry with high angular resolution for functional materials with phase coexistences. The weak unit-cell distortions in BCZT can only be resolved with the MAD. At the same time, the combination with the stroboscopic data-acquisition setup allows time resolutions in the range of microseconds (Choe et al., 2015).

\section{Structural characterization of STN and SZN \\ 5.1. Experimental}

Data were collected at a wavelength of $\lambda=0.2074426$ (4) $\AA$. Two-dimensional data were collected with a 16-inch ( $\sim 409.6 \mathrm{~mm})$ 2D flat panel detector of the XRD 1621N ES Series (PerkinElmer) with $2048 \times 2048$ pixels and a pixel size of $200 \mu^{2}$. The sample distance was $2513 \mathrm{~mm}$ in order to achieve high resolution. To meet a high signal-to-noise ratio, the exposure time was $60 \mathrm{~s}$. Details about the setup can be found elsewhere (Herklotz et al., 2013).

High-resolution data were collected with the MAD in the range $0.5^{\circ} \leq 2 \theta \leq 12.5^{\circ}$. In order to have high statistics and an ideal signal-to-noise ratio even for low-intensity reflections, the full pattern was merged from $3^{\circ}$ stretches of every channel 
(see the supporting information; Lee, Shi, Kumar, Hoffman, Etter, Checchia, Lemos da Silva et al., 2020). This results in high counting statistics in the angular range $2.5^{\circ} \leq 2 \theta \leq 10.5^{\circ}$. In the low and high angular ranges and depending on the $2 \theta$ range, only one single pattern or two patterns were measured. Data were collected with an angular step width of $\Delta 2 \theta=0.0005^{\circ}$ and an exposure time of $7.5 \mathrm{~s}$ per point.

Rietveld refinement was performed with the program package FullProf (RodríguezCarvajal, 1993). The structure models consisted of three phases of STN. The main phase, $\mathrm{Sr}_{3} \mathrm{Nb}_{4} \mathrm{TiO}_{15}$, crystallizes with space group Pna2 1 (Whittle \& Schmid, 2014; Whittle et al., 2017). Additionally, two impurity phases were identified. A cubic perovskite phase of $\mathrm{SrNb}_{0.8} \mathrm{Ti}_{0.2} \mathrm{O}_{3}$ with space group $P m \overline{3} m$ and a complex phase $\mathrm{Sr}_{5} \mathrm{Nb}_{4} \mathrm{TiO}_{17}$ with space group Pnnm (Drews et al., 1996). The instrumental broadening was determined by a Rietveld fit of a high-resolution measurement recorded at ambient temperature of the standard reference material $\mathrm{LaB}_{6}$ (SRM 660a, NIST) for X-ray measurements. The profile function was described using the Thompson-CoxHastings pseudo-Voigt model (Thompson et al., 1987). Lattice parameters, background, scale factors, zero shift for the MAD data and the overall Debye-Waller factor were refined. All other structural information such as atomic positions or individual Debye-Waller factors were kept from literature values. The refinement with the MAD and $2 \mathrm{D}$ data was performed simultaneously with a single structure model of three phases in order to combine the high statistics from 2D and the high angular resolution from the MAD.

\subsection{Results and discussion}

Fig. 7 shows a comparison of the X-ray diffraction patterns of STN. While Fig. 7(a) shows the high-resolution diffraction pattern collected with the MAD, Fig. 7(b) shows the diffraction pattern of the same sample collected with the 2D Perkin Elmer detector at a high-resolution distance to the sample of $2513 \mathrm{~mm}$. The difference plot in Fig. 7(b) demonstrates that the structure model can explain the observed intensities very well. However, the difference plot in Fig. 7(a) exhibits some strong deviations between the calculated and the observed intensities. A closer look at some characteristic reflections with exceptional deviation, plotted in the insets, shows that the deviations originate from profile mismatches. Most prominent is the profile mismatch for the 004 reflection, shown in inset (I) of Fig. 7(a), which cannot be observed with the 2D detector [Fig. 7(a), inset (II)]. This reflection shows a pronounced asymmetry towards lower angles. This form of asymmetry might originate from stacking faults owing to the plate-like
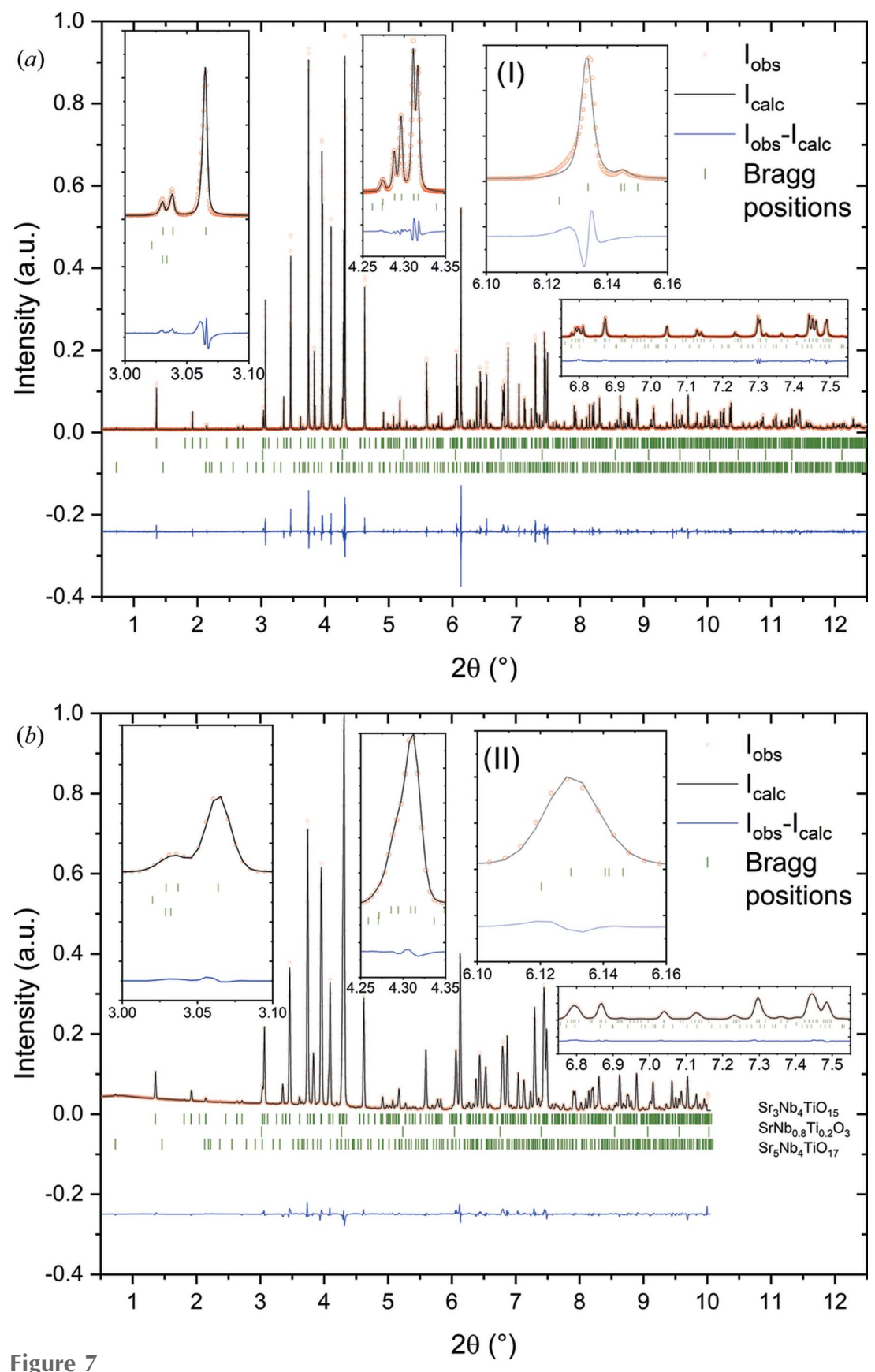

Rietveld refinements with X-ray diffraction patterns of STN, measured with $(a)$ the highresolution MAD and $(b)$ the 2D Perkin Elmer detector in high-resolution mode at a sample detector distance of $2513 \mathrm{~mm}$. The diffraction data reveal two impurity phases: $\mathrm{SrNb}_{0.8} \mathrm{Ti}_{0.2} \mathrm{O}_{3}[1.02(8) \%]$ and $\mathrm{Sr}_{5} \mathrm{Nb}_{4} \mathrm{TiO}_{17}[1.45(19) \%]$. The insets show magnifications of a range of individual reflections. The 004 reflection in inset (I) of the high-resolution MAD data reveals an asymmetry, which may result from stacking faults along $00 l$, which can only be detected with the highest angular resolution and not with the 2D detector as shown in inset (II). Red dots indicate measured intensities, black lines indicate the calculated diffraction pattern from the structure model, blue lines indicate the difference between measured and calculated intensities, and green tick marks indicate reflection positions of the respective phases. $\lambda=0.2074426$ (4) $\AA$. 
structure of the main phase (Estevez-Rams et al., 2003). Octahedra in subsequent layers of this structure along the $c$ axis are constraint to tilt in the opposite sense reducing the likelihood of stacking faults (Whittle et al., 2015, 2018; Campbell et al., 2018). These might, however, originate at possible cation defect sites. A detailed microstructural analysis
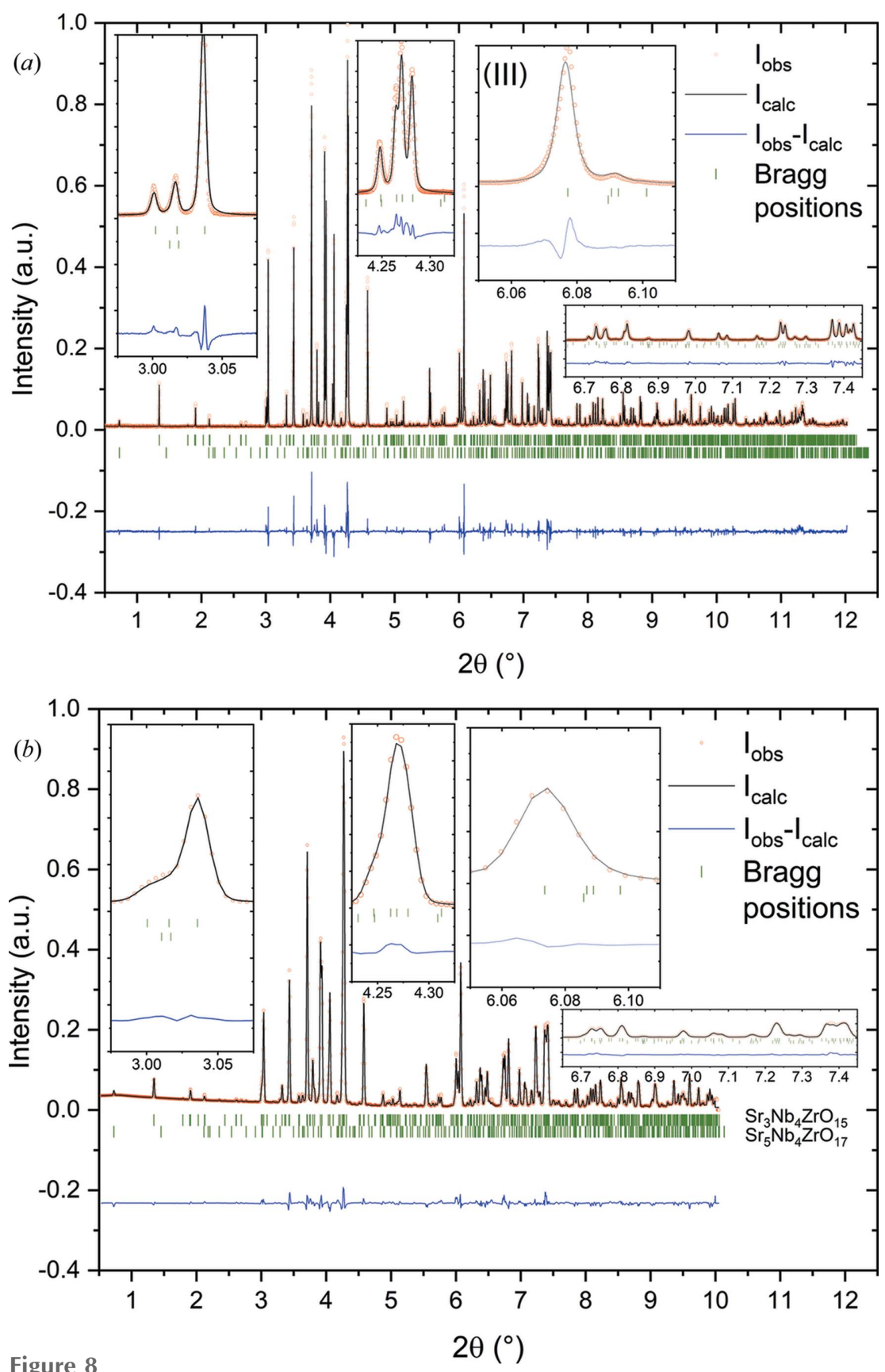

Figure 8

Rietveld refinements with X-ray diffraction patterns of SZN, measured with $(a)$ the highresolution MAD and $(b)$ the $2 \mathrm{D}$ Perkin Elmer detector in high-resolution mode at a sample detector distance of $2513 \mathrm{~mm}$. The diffraction data reveal one impurity phase: $\mathrm{SrNb}_{0.8} \mathrm{Zr}_{0.2} \mathrm{O}_{3}$ [4.52 (25)\%]. The insets show magnifications of a range of individual reflections. Compared with STN, the asymmetry of the 004 reflection in inset (III) of the high-resolution MAD data is much less pronounced, which indicates only small amounts of stacking faults along 00l. Red dots indicate measured intensities, black lines indicate the calculated diffraction pattern from the structure model, blue lines indicate the difference between measured and calculated intensities, and green tick marks indicate reflection positions of the respective phases. $\lambda=0.2074426$ (4) $\AA$. of this complex material may be able to shed light on a possible mechanism but is beyond the scope of the work

8 shows a comparison of the $\mathrm{X}$-ray diffraction patterns of SZN, measured with the MAD and the 2D detector. The structure models are similar to STN with slight differences in lattice parameters (Whittle et al., 2020). The difference curve of the 2D data in Fig. 8(b) shows an agreement similar to that shown in Fig. 7(b), which indicates an equally well fitting structure model. However, a closer look at the MAD data in Fig. 8(a) reveals a significantly worse fit. Although the asymmetry of the 004 reflection cannot be seen very well anymore [Fig. 8(a), inset (III)], the general mismatch of the profile is still the main reason for the difference between observed and calculated intensities. For this sample, the impurity phase content is higher with $\sim 4.5 \mathrm{wt} \%$. The impurity phase was found to be $\mathrm{Sr}_{5} \mathrm{Nb}_{4} \mathrm{ZrO}_{17}$ in analogy with STN. In this phase, titanium was substituted by zirconium. However, this phase is not known in the literature nor in any crystal-structure database. The fact that it explains all additional reflections with the modified structure model of $\mathrm{Sr}_{5} \mathrm{Nb}_{4} \mathrm{TiO}_{17}$ from the literature (Drews et al., 1996) strongly suggests the existence of this compound. Similar to STN, the proof of existence of this impurity phase was only possible with the combination of MAD and 2D data.

The comparison of the MAD data with the 2D data reveals that, even in the high-resolution position, the $2 \mathrm{D}$ detector cannot resolve the fine reflection splitting of STN or SZN. Especially in the range around $2 \theta=4.3^{\circ}$, the MAD can resolve five reflections of STN from three phases while the $2 \mathrm{D}$ detector only shows a slightly asymmetric single reflection. The combination of MADs and 2D detectors proves to be a strong tool for solving mixed structures from powder-diffraction data.

\section{In situ stroboscopic investigation of the field-induced processes in BCZT}

\subsection{Experimental}

Data were collected at a wavelength of $\lambda=$ 0.2072066 (4) $\AA$. The measurements were performed with the custom-built stroboscopic data system MAD-STROBO for timeresolved X-ray diffraction experiments on time scales down to 10 ns (Choe et al., 2015). Powder X-ray diffraction patterns were taken in transmission geometry with the electric 
field parallel to the scattering vector. The structural response was measured with the highest possible resolution of the beamline P02.1 with the MAD. The maximum time resolution of the diffraction signal (defined by the width of the time channel) was $10 \mathrm{~ns}$ - every 100 adjacent channels were binned to reduce the time resolution to $1 \mu \mathrm{s}$.

The investigated sample $0.6 \mathrm{BaZr}_{0.2} \mathrm{Ti}_{0.8} \mathrm{O}_{3}-$ $0.4 \mathrm{Ba}_{0.7} \mathrm{Ca}_{0.3} \mathrm{TiO}_{3}$ (Acosta et al., 2015) has dimensions of $1 \mathrm{~mm} \times 1 \mathrm{~mm} \times 5 \mathrm{~mm}$ with electrodes on two opposing long sides that are connected to a high-voltage supply. For this sample, $\mu R=1.80$ at $60 \mathrm{keV}$, which leads to a transmitted intensity of $2.75 \%$. At usual high-resolution beamlines operating at the highest possible energies of $40 \mathrm{keV}, \mu R=5.35$ affords a transmitted intensity of $0.002 \%$. At $30 \mathrm{keV}$, which marks the highest possible energy for silicon-based-strip and 2D detectors, $\mu R=8.40$ affords a transmitted intensity of $<0.0001 \%$. This shows that these in situ high-resolution measurements are only feasible with a combination of high photon energy and a MAD.

\subsection{Results and discussion}

The aim of this experiment was to investigate the structural changes in $0.6 \mathrm{BaZr}_{0.2} \mathrm{Ti}_{0.8} \mathrm{O}_{3}-0.4 \mathrm{Ba}_{0.7} \mathrm{Ca}_{0.3} \mathrm{TiO}_{3} \quad$ (BZT40BCT) ferroelectric ceramics in situ, induced by a unipolar alternating electric field. The BZT $-x$ BCT system exhibits a complex phase diagram with rhombohedral, orthorhombic and tetragonal structures below the Curie temperature (Keeble et al., 2013). The Curie temperatures for the compositions around the phase boundaries of these phases are relatively close to room temperature in the range between $60^{\circ} \mathrm{C}$ and $90^{\circ} \mathrm{C}$. Therefore, the BZT $-x \mathrm{BCT}$ system is important for electrocaloric applications. However, the unit-cell distortions are weak, which demands for high angular resolution to evaluate crystallographic distortions. Recently, a strong electrocaloric effect was reported for BZT-35BCT (Sanlialp et al., 2015). This effect peaks around the Curie temperature, which is $\sim 70^{\circ} \mathrm{C}$ for the composition BZT-40BCT. In addition, complex polarization dynamics were demonstrated in these materials as a function of temperature, which largely determine the piezoelectric activity (Zhukov et al., 2015). In order to understand these functional properties and correlate them with the structure of the materials, we performed detailed structural investigations above the Curie temperature.

While ferroelectrics are expected to exhibit cubic structure above the Curie temperature, we recently showed in the system BNT $-x$ BT that an applied electric field can induce a paraelectric ferroelectric phase transformation, even several degrees above the Curie temperature (Wang et al., 2014). In order to investigate this behaviour in BZT- $x \mathrm{BCT}$ and correlate structural distortions with functional properties, we performed stroboscopic high-resolution powder diffraction above the Curie temperature. The angular resolution at the physical limit allows resolving of even the slightest lattice distortions. Together with the stroboscopic technique, we can thus access fine responses at timescales in the range of microseconds in order to elucidate the electrocaloric phenomenon.

Figs. $9(a)$ and $9(b)$ show the time-resolved powder-diffraction profiles of the 111 and 200 reflections under a $10 \mathrm{kHz}$ electric field of $0.3 \mathrm{kV} \mathrm{mm}^{-1}$. The contour plots directly show pronounced changes to the applied electric field. While the strongest changes of the 111 reflection occur directly after switching on and off the field, the response of the 200 reflection is significantly delayed. The increasing reflection intensities indicate a change of crystal structure or an increase of order [i.e. a decrease of distributions of lattice distortions as found in domain structures (Boysen, 2007)] as a function of the applied electric field. While the 111 reflection can display

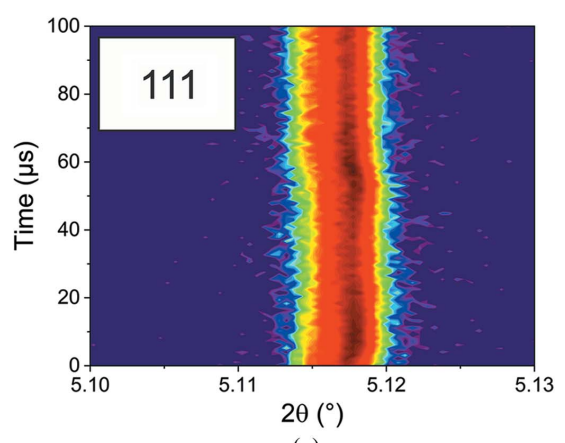

(a)

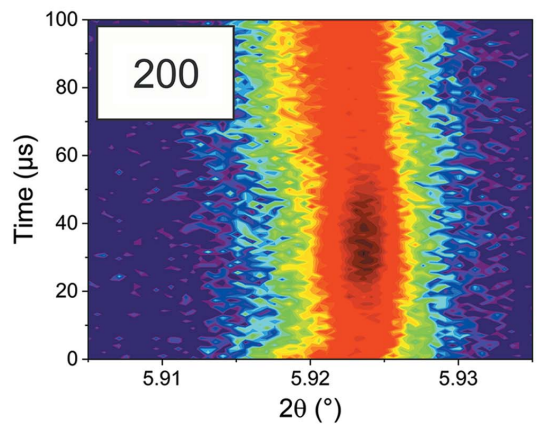

(b)

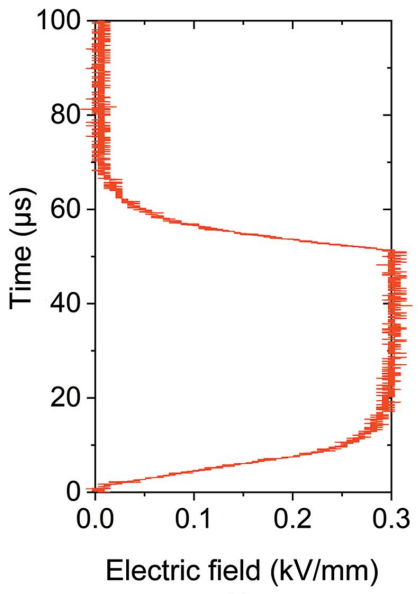

(c)

Figure 9

Stroboscopic high-resolution powder diffraction with $10 \mathrm{kHz}$ and $0.3 \mathrm{kV} \mathrm{mm}^{-1}$ with a time resolution of $1 \mu \mathrm{s}$ of BCT-40BZT. Contour plots of the (a) 111 and (b) 200 reflections and (c) the corresponding electric field profile. 
a splitting owing to orthorhombic (Amm2) or rhombohedral $(R 3 m)$ distortion, the 200 reflection indicates orthorhombic $(A m m 2)$ or tetragonal $(P 4 m m)$ distortion. In this experiment, the electric field is perpendicular to the incident beam. Therefore, we measure lattice planes that are perpendicular to the electric field and can monitor the piezoelectric effect along the electric field.

Since the applied electric field is a unidirectional force, it induces a preferred orientation and growth of domains with polar directions close to the field direction (Acosta et al., 2016). The growth of domains with a specific orientation decreases the amount of domain walls and thus the distribution of lattice distortions (Boysen, 2007). As reported by Jin et al. (2003), fine domain structures in the range of nanometres near phase boundaries can result from conformal miniaturization. With applied field, the authors could show in a relaxor ferroelectric system that the application in such a system leads to discontinuous changes in lattice parameters. This ordering induced by the anisotropy of the electric field together with the very low tolerance level concerning Bragg's law can result in a change in integrated intensity. This is because it involves an increase in scattering volume that fulfils the diffraction condition. Therefore, the observations in Fig. 9 can be explained by phase transformations or domain ordering.

Upon field application ( $t=0 \mu \mathrm{s})$, the integrated intensity of the reflections increases with the same slope as the electric field. Therefore, the kinetics of this response is at least faster than $10 \mu \mathrm{s}$. In this time range, the electric field may induce a phase transformation or the precipitation of domains. At $t=$ $15 \mu$ s when the maximum field is reached, the intensity of the 111 reflection decreases again, while the intensity of the 200 reflection increases. Since both intensities are inversely correlated, this indicates a complex structural response of the material.

At $t=40 \mu \mathrm{s}$, the 200 intensity decreases again even though the maximum electric field is still applied. At the same time, the 111 intensity increases back to the maximum reached at the beginning of the field application. This time range might indicate competing strain mechanisms with resonant elastic responses, especially because the 111 response exhibits an exact symmetrical shape beyond the moment of switching off the field. In contrast to the instant response at the rising edge, the response at the falling edge is significantly delayed. This might indicate resonant vibrations owing to the competing structural strain mechanisms.

Fig. 10 depicts the same reflections on 3D contour plots. This representation illustrates the complex and contrarious structural response of the material as a function of time and thus applied electric field. The intensity of the 200 reflection increases dramatically after applying the field and shows pronounced kinetics. At the same time the intensity of the 111 reflection decreases and both intensities follow an opposite behaviour. This again shows the structural connection and points towards a field-induced phase transformation. Since the 200 reflection is significantly broader, it indicates a tetragonal distortion.

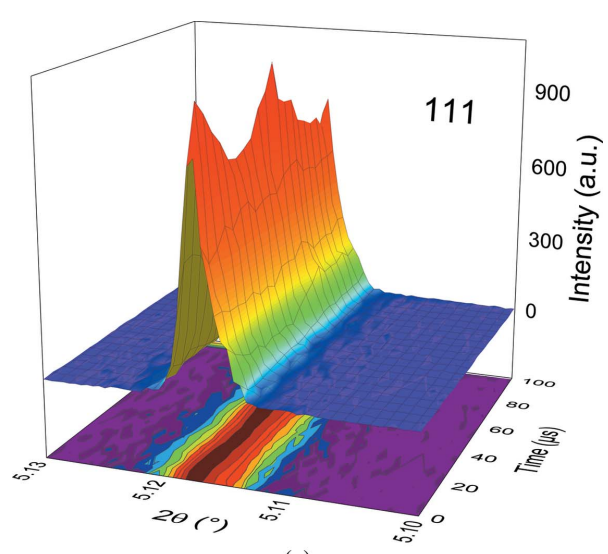

(a)

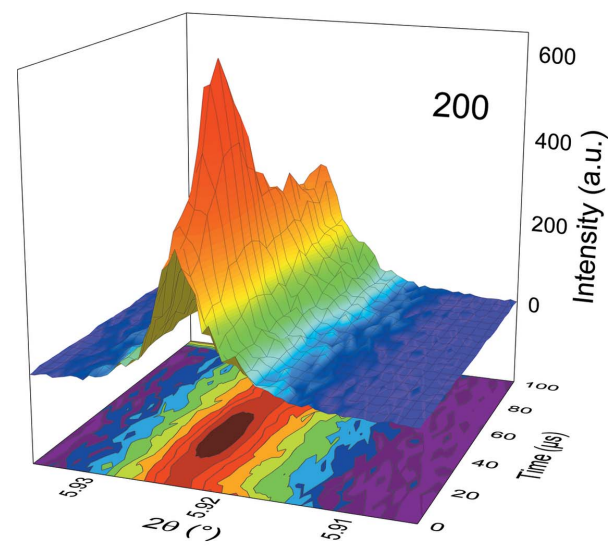

(b)

Figure 10

3D contour plots of $(a)$ the 111 and (b) 200 reflections.

\section{Conclusions}

The structural investigation of STN and SZN reveals that, even in the high-resolution position, the 2D detector cannot resolve the fine distortion of the structure. Some reflections exhibit a fivefold splitting, while the $2 \mathrm{D}$ detector only shows a slightly asymmetric single reflection. We demonstrate that it is possible to attain a significant reduction in measuring time with a remarkable resolution using a high-resolution MAD. Stitching together $3^{\circ}$ slices of the ten individual channels of the MAD allowed a significant reduction in measuring time for this high-resolution detector and resulted in a good fit, apart from the profile mismatches owing to the real structure of the sample. The fit shows that small quantities of $\sim 1-2 \mathrm{wt} \%$ can still be detected. This also demonstrates the high signal-tonoise ratio and high accuracy in detected intensities. Without the MAD data, it would not have been possible to identify the impurity phases, owing to the strong overlap within the 2D dataset. For the case of SZN, even a formerly unknown compound could be identified. The combination of MADs and $2 \mathrm{D}$ detectors proves to be a strong tool for solving mixed structures from powder-diffraction data.

The stroboscopic experiment with applied electric field shows a complex range of responses above the Curie temperature. Only the combination of high angular resolution at the physical limit with the highest brilliance of the 
synchrotron radiation and a time resolution in the range of microseconds is able to reveal these field-induced processes. This sophisticated experiment demonstrates the possibilities for next-generation materials characterization. The elucidation of competing structural strain mechanisms above the Curie temperature with significantly different time scales are of great importance for the understanding of functional ceramics. These experiments and the MAD become particularly relevant owing to the large absorption of these materials. A detailed characterization of the kinetics of the individual strain mechanisms allow optimizing the efficiency of actuators and electrocaloric applications. Since these complex responses on a microsecond time scale were formerly unknown, this characterization technique has an extraordinary impact on research and development of functional piezoceramics.

\section{Acknowledgements}

We acknowledge DESY (Hamburg, Germany), a member of the Helmholtz Association HGF, for the provision of experimental facilities. Parts of this research were carried out at beamline P02.1. We thank Helmholtz-Zentrum Berlin (HZB) for the allocation of synchroton radiation beam time. Open access funding enabled and organized by Projekt DEAL.

\section{Funding information}

The research leading to these results received funding from the BMBF (Bundesministerium fuer Bildung und Forschung) (grant Nos. 05K10ODA and 05K13VK1) and the DFG (Deutsche Forschungsgemeinschaft) (grant No. HI 1867/1-1).

\section{References}

Acosta, M., Khakpash, N., Someya, T., Novak, N., Jo, W., Nagata, H., Rossetti, G. A. Jr \& Rödel, J. (2015). Phys. Rev. B, 91, 104108.

Acosta, M., Schmitt, L. A., Cazorla, C., Studer, A. J., Zintler, A., Glaum, J., Kleebe, H.-J., Donner, W., Hoffman, M., Rödel, J. \& Hinterstein, M. (2016). Sci. Rep. 6, 28742.

Billinge, S. J. L. (2019). Philos. Trans. A Math. Phys. Eng. Sci. 377, 20190037.

Boysen, H. (2007). J. Phys. Condens. Matter, 19, 275206.

Buras, B. \& Christensen, F. (1981). HASYLAB Annual Report, p. 101. HASYLAB, Hamburg, Germany.

Campbell, B., Howard, C. J., Averett, T. B., Whittle, T. A., Schmid, S., Machlus, S., Yost, C. \& Stokes, H. T. (2018). Acta Cryst. A74, 408-424.

Choe, H., Gorfman, S., Hinterstein, M., Ziolkowski, M., Knapp, M., Heidbrink, S., Vogt, M., Bednarcik, J., Berghäuser, A., Ehrenberg, H. \& Pietsch, U. (2015). J. Appl. Cryst. 48, 970-974.

Coelho, A. A. (2018). J. Appl. Cryst. 51, 210-218.

Cox, D. E., Hastings, J. B., Cardoso, L. P. \& Finger, L. W. (1986). Mater. Sci. Forum, 9, 1-20.

Cox, D. E., Hastings, J. B., Thomlinson, W. \& Prewitt, C. T. (1983). Nucl. Instrum. Methods Phys. Res. 208, 573-578.

Cullity, B. D. \& Stock, S. R. (2001). Elements of X-ray Diffraction. New York: Prentice Hall.

Daniels, J. E., Cozzan, C., Ukritnukun, S., Tutuncu, G., Andrieux, J., Glaum, J., Dosch, C., Jo, W. \& Jones, J. L. (2014). J. Appl. Phys. 115, 224104.
Dejoie, C., Coduri, M., Petitdemange, S., Giacobbe, C., Covacci, E., Grimaldi, O., Autran, P.-O., Mogodi, M. W., Šišak Jung, D. \& Fitch, A. N. (2018). J. Appl. Cryst. 51, 1721-1733.

Dippel, A.-C., Liermann, H.-P., Delitz, J. T., Walter, P., SchulteSchrepping, H., Seeck, O. H. \& Franz, H. (2015). J. Synchrotron Rad. 22, 675-687.

Drews, A. R., Wong-Ng, W., Roth, R. S. \& Vanderah, T. A. (1996). Mater. Res. Bull. 31, 153-162.

Ehrenberg, H., Hinterstein, M., Senyshyn, A. \& Fuess, H. (2019). International Tables for Crystallography, Vol. H, pp. 174-188. Chester: International Union of Crystallography.

Ehrenberg, H., Knapp, M., Hartmann, T., Fuess, H. \& Wroblewski, T. (2000). J. Appl. Cryst. 33, 953-957.

Ehrenberg, H., Senyshyn, A., Hinterstein, M. \& Fuess, H. (2013). Modern Diffraction Methods, Vol. edited by E. J. Mittemeijer \& U. Welzel, pp. 491-517. Weinheim: Wiley-VCH.

Estevez-Rams, E., Leoni, M., Scardi, P., Aragon-Fernandez, B. \& Fuess, H. (2003). Philos. Mag. 83, 4045-4057.

Fauth, F., Peral, I., Popescu, C. \& Knapp, M. (2013). Powder Diffr. 28, S360-S370.

Finger, L. W., Cox, D. E. \& Jephcoat, A. P. (1994). J. Appl. Cryst. 27, 892-900.

Fitch, A. N. (2004). J. Res. Natl Inst. Stand. Technol. 109, 133.

Geiger, P. T., Clemens, O., Khansur, N. H., Hinterstein, M., Sahini, M. G., Grande, T., Tung, P., Daniels, J. E. \& Webber, K. G. (2017). Solid State Ion. 300, 106-113.

Geiger, P. T., Khansur, N. H., Riess, K., Martin, A., Hinterstein, M. \& Webber, K. G. (2018). J. Appl. Phys. 123, 075104.

Gozzo, F., De Caro, L., Giannini, C., Guagliardi, A., Schmitt, B. \& Prodi, A. (2006). J. Appl. Cryst. 39, 347-357.

Gozzo, F., Schmitt, B., Bortolamedi, T., Giannini, C., Guagliardi, A., Lange, M., Meister, D., Maden, D., Willmott, P. \& Patterson, B. D. (2004). J. Alloys Compd. 362, 206-217.

Hastings, J. B., Thomlinson, W. \& Cox, D. E. (1984). J. Appl. Cryst. 17, $85-95$.

Herklotz, M., Scheiba, F., Hinterstein, M., Nikolowski, K., Knapp, M., Dippel, A.-C., Giebeler, L., Eckert, J. \& Ehrenberg, H. (2013). J. Appl. Cryst. 46, 1117-1127.

Hinterstein, M., Lee, K.-Y., Esslinger, S., Glaum, J., Studer, A. J., Hoffman, M. \& Hoffmann, M. J. (2019). Phys. Rev. B, 99, 174107.

Hinterstein, M., Mgbemere, H. E., Hoelzel, M., Rheinheimer, W., Adabifiroozjaei, E., Koshy, P., Sorrell, C. C. \& Hoffman, M. (2018). J. Appl. Cryst. 51, 670-678.

Hodeau, J.-L., Bordet, P., Anne, M., Prat, A., Fitch, A. N., Dooryhee, E., Vaughan, G. \& Freund, A. K. (1998). Proc. SPIE, 3448, 353.

Horst, A., Berghaeuser, A., Hinterstein, M. \& Knapp, M. (2013). DE Patent 102012220124B3.

Jin, Y. M., Wang, Y. U., Khachaturyan, A. G., Li, J. F. \& Viehland, D. (2003). Phys. Rev. Lett. 91, 197601.

Keeble, D. S., Benabdallah, F., Thomas, P. A., Maglione, M. \& Kreisel, J. (2013). Appl. Phys. Lett. 102, 092903.

Knapp, M., Baehtz, C., Ehrenberg, H. \& Fuess, H. (2004). J. Synchrotron Rad. 11, 328-334.

Lausi, A., Polentarutti, M., Onesti, S., Plaisier, J. R., Busetto, E., Bais, G., Barba, L., Cassetta, A., Campi, G., Lamba, D., Pifferi, A., Mande, S. C., Sarma, D. D., Sharma, S. M. \& Paolucci, G. (2015). Eur. Phys. J. Plus, 130, 43.

Lee, K.-Y., Shi, X., Kumar, N., Hoffman, M., Etter, M., Checchia, S., Lemos da Silva, L., Seifert, D. \& Hinterstein, M. (2020). Materials (Basel). Submitted.

Lee, K.-Y., Shi, X., Kumar, N., Hoffman, M., Etter, M., Checchia, S., Winter, J., Lemos da Silva, L., Seifert, D. \& Hinterstein, M. (2020). Materials (Basel). 13, 1054.

Lee, K.-Y., Shi, X., Kumar, N., Hoffman, M., Etter, M., Winter, J., Lemos da Silva, L., Seifert, D. \& Hinterstein, M. (2020). Appl. Phys. Lett. 116, 182902. 
Lee, P. L., Shu, D., Ramanathan, M., Preissner, C., Wang, J., Beno, M. A., Von Dreele, R. B., Ribaud, L., Kurtz, C., Antao, S. M., Jiao, X. \& Toby, B. H. (2008). J. Synchrotron Rad. 15, 427-432.

Liu, L., Knapp, M., Ehrenberg, H., Fang, L., Fan, H., Schmitt, L. A., Fuess, H., Hoelzel, M., Dammak, H., Thi, M. P. \& Hinterstein, M. (2017). J. Eur. Ceram. Soc. 37, 1387-1399.

Liu, L., Knapp, M., Ehrenberg, H., Fang, L., Schmitt, L. A., Fuess, H., Hoelzel, M. \& Hinterstein, M. (2016). J. Appl. Cryst. 49, 574584.

Liu, L., Knapp, M., Schmitt, L. A., Ehrenberg, H., Fang, L., Fuess, H., Hoelzel, M. \& Hinterstein, M. (2016). EPL (Europhysics Letters), 114, 47011.

Liu, Q., Zhang, Y., Gao, J., Zhou, Z., Yang, D., Lee, K.-Y., Studer, A., Hinterstein, M., Wang, K., Zhang, X., Li, L. \& Li, J.-F. (2020). Natl Sci. Rev. 7, 355-365.

Masson, O., Dooryhée, E., Cheary, R. W. \& Fitch, A. N. (2001). Mater. Sci. Forum, 378-381, 300-307.

Mgbemere, H. E., Schneider, G. A., Schmitt, L. A. \& Hinterstein, M. (2017). J. Ceram. Sci. Technol. 8, 45-52.

Parrish, W., Hart, M., Erickson, C. G., Masciocchi, N. \& Huang, T. C. (1985). Adv.X-ray Anal. 29, 243-250.

Parrish, W., Hart, M. \& Huang, T. C. (1986). J. Appl. Cryst. 19, 92100.

Patterson, B. D., Brönnimann, C., Maden, D., Gozzo, F., Groso, A., Schmitt, B., Stampanoni, M. \& Willmott, P. R. (2005). Nucl. Instrum. Methods Phys. Res. B, 238, 224-228.

Pawley, G. S. (1981). J. Appl. Cryst. 14, 357-361.

Peral, I., McKinlay, J., Knapp, M. \& Ferrer, S. (2011). J. Synchrotron Rad. 18, 842-850.

Ren, Y. \& Zuo, X. (2018). Small Methods, 2, 1800064.

Riess, K., Geiger, P. T., Khansur, N. H., Steiner, S., Frömling, T., Hinterstein, M. \& Webber, K. G. (2019). Materialia, 6, 100297.

Rodríguez-Carvajal, J. (1993). Physica B, 192, 55-69.

Rouquette, J., Hinterstein, M., Haines, J., Papet, P., Knap, M., Glaum, J. \& Fuess, H. (2012). Acta Cryst. A68, s94.

Sanlialp, M., Shvartsman, V. V., Acosta, M., Dkhil, B. \& Lupascu, D. C. (2015). Appl. Phys. Lett. 106, 062901.

Schader, F. H., Wang, Z., Hinterstein, M., Daniels, J. E. \& Webber, K. G. (2016). Phys. Rev. B, 93, 134111.

Schmitt, L. A., Kungl, H., Hinterstein, M., Riekehr, L., Kleebe, H.-J., Hoffmann, M. J., Eichel, R.-A. \& Fuess, H. (2013). 2013 Joint IEEE International Symposium on Applications of Ferroelectric and Workshop on Piezoresponse Force Microscopy (ISAF/PFM), pp. 149-151. IEEE.
Shi, X., Ghose, S. \& Dooryhee, E. (2013). J. Synchrotron Rad. 20, 234-242.

Staub, U., Shi, M., O’Conner, A. G., Kramer, M. J. \& Knapp, M. (2001). Phys. Rev. B, 63, 134522.

Staub, U., Soderholm, L., Wasserman, S., Conner, A. G. O., Kramer, M. J., Patterson, B. D., Shi, M. \& Knapp, M. (2000). Phys. Rev. B, 61, 1548-1554.

Sutter, J. P., Chater, P. A., Hillman, M. R., Keeble, D. S., Tucker, M. G. \& Wilhelm, H. (2016). AIP Conf. Proc. 1741, 040005.

Thompson, P., Cox, D. E. \& Hastings, J. B. (1987). J. Appl. Cryst. 20, 79-83.

Thompson, S. P., Parker, J. E., Potter, J., Hill, T. P., Birt, A., Cobb, T. M., Yuan, F. \& Tang, C. C. (2009). Rev. Sci. Instrum. 80, 075107. Toraya, H. (2009). J. Appl. Cryst. 42, 485-489.

Toraya, H., Hibino, H. \& Ohsumi, K. (1996). J. Synchrotron Rad. 3, $75-83$.

Vaughan, G. B. M., Baker, R., Barret, R., Bonnefoy, J., Buslaps, T., Checchia, S., Duran, D., Fihman, F., Got, P., Kieffer, J., Kimber, S. A. J., Martel, K., Morawe, C., Mottin, D., Papillon, E., Petitdemange, S., Vamvakeros, A., Vieux, J.-P. \& Di Michiel, M. (2020). J. Synchrotron Rad. 27, 515-528.

Wallwork, K. S., Kennedy, B. J. \& Wang, D. (2007). AIP Conf. Proc. 879, 879-882.

Wang, J., Toby, B. H., Lee, P. L., Ribaud, L., Antao, S. M., Kurtz, C., Ramanathan, M., Von Dreele, R. B. \& Beno, M. A. (2008). Rev. Sci. Instrum. 79, 085105.

Wang, Z., Webber, K. G., Hudspeth, J. M., Hinterstein, M. \& Daniels, J. E. (2014). Appl. Phys. Lett. 105, 161903.

Whittle, T. A., Brant, W. R., Hester, J. R., Gu, Q. \& Schmid, S. (2017). Dalton Trans. 46, 7253-7260.

Whittle, T. A. \& Schmid, S. (2014). Powder Diffr. 29, S15-S18.

Whittle, T. A., Schmid, S. \& Howard, C. J. (2015). Acta Cryst. B71, 342-348.

Whittle, T. A., Schmid, S. \& Howard, C. J. (2018). Acta Cryst. B74, 742-744.

Whittle, T., Lu, T., Blanchard, P., Hester, J., Gu, Q., Liu, Y. \& Schmid, S. (2020). CrystEngComm, 22, 4994-5001.

Yang, T. Y., Wen, W., Yin, G. Z., Li, X. L., Gao, M., Gu, Y. L., Li, L., Liu, Y., Lin, H., Zhang, X. M., Zhao, B., Liu, T. K., Yang, Y. G., Li, Z., Zhou, X. T. \& Gao, X. Y. (2015). Nucl. Sci. Tech. 26, 020101.

Yavuz, M., Knapp, M., Indris, S., Hinterstein, M., Donner, W. \& Ehrenberg, H. (2015). J. Appl. Cryst. 48, 1699-1705.

Zhukov, S., Acosta, M., Genenko, Y. A. \& von Seggern, H. (2015). J. Appl. Phys. 118, 134104. 